\title{
Reflets
}

Revue d'intervention sociale et communautaire

\section{GRELL, Paul (2015). Adolescence et suicide, Paris : BERG International éditeurs, 216 p.}

\section{Lise Savoie}

Volume 22, numéro 2, automne 2016

URI : https://id.erudit.org/iderudit/1038978ar

DOI : https://doi.org/10.7202/1038978ar

Aller au sommaire du numéro

\section{Éditeur(s)}

Reflets, Revue d'intervention sociale et communautaire

ISSN

1203-4576 (imprimé)

1712-8498 (numérique)

Découvrir la revue

Citer ce compte rendu

Savoie, L. (2016). Compte rendu de [GRELL, Paul (2015). Adolescence et suicide, Paris : BERG International éditeurs, 216 p.] Reflets, 22(2), 222-225.

https://doi.org/10.7202/1038978ar d'utilisation que vous pouvez consulter en ligne.

https://apropos.erudit.org/fr/usagers/politique-dutilisation/ 


\section{Adolescence et suicide}

GRELL, Paul (2015). Paris : BERG International éditeurs, 216 p.

\section{par Lise Savoie}

Professeure agrégée,

École de travail social, Faculté des arts et des sciences sociales,

Université de Moncton

Commençons par dire qu'Adolescence et suicide est le troisième livre du professeur Paul Grell qui porte sur la précarité des modes de vie. Dans ce dernier livre, basé sur les récits de vie de jeunes francophones du Nouveau-Brunswick, l'auteur s'intéresse à la parole des jeunes comme experts de leur situation. Non seulement il nous conduit vers les ombres et les eaux troubles des expériences de vie précaires des jeunes, mais également il nous propose de réfléchir aux formes de « débrouillardise sociale » dont font preuve ces jeunes.

Ce livre propose de penser le suicide des jeunes à l'extérieur des explications de "suicidologues ", que l'auteur juge trop simplistes et qui lient l'acte de la mort volontaire à un problème de santé mentale. Or, si la personne n'a pas d'antécédent de santé mentale ou encore n'a pas démontré de signe attribuable à la santé mentale, alors on est devant une incompréhension totale, un acte incompris, une anomalie ou encore un acte anodin, comme le dit l'auteur. Celui-ci se demande d'ailleurs si cette manière de concevoir, de comprendre ou encore d'interpréter l'acte suicidaire n'est pas attribuable à "l'étroitesse d'un regard posé ». Dans Adolescence et suicide, Paul Grell exprime d'emblée l'idée que le suicide des jeunes est difficilement explicable et que sa compréhension doit avant tout passer par un regard sur les pratiques quotidiennes des jeunes. Pour ne pas que l'acte suicidaire "se perde dans l'indifférence », ce livre tient simplement à donner une voix aux jeunes qui ont côtoyé, vu ou frôlé cette mort volontaire. En fait, qu'en pensent ces jeunes? Dès la première page du livre, l'auteur pose également une question fondamentale à cet ouvrage : "Comment comprendre que nous ne comprenons pas ou si peu l'avènement des suicides de jeunes? » Comme le souligne si bien l'auteur, « c'est par les faits et gestes les plus ordinaires qu'il faut d'abord tenter de cerner un être du point de vue phénoménologique, comme si on crayonnait à grands traits les valeurs profondes de ce qui fait son humanité ». 
Le livre est divisé en deux parties. La première intitulée Les enfants du chaos comporte quatre chapitres qui mettent en avant-scène des expériences mortiferes que vivent les jeunes. L'auteur s'interroge, par exemple, dans le premier chapitre, sur « les petites mises à mort » que les jeunes vivent. Il s'agit d'expériences de vie quotidienne dans lesquelles se confrontent des situations de vie difficiles avec le vécu d'amis qui s'enlèvent la vie. Ce sont des récits croisés, les leurs et ceux de proches, qui renvoient au mal de vivre dans un monde qui leur est hostile. Malgré tout, ces jeunes choisissent la vie, comme il en est question au deuxième chapitre. Parfois en adoptant des pratiques à risque, certains réinventent leur vie autrement. Pour qu'ils puissent s'en sortir, la fuite vers d'autres lieux devient leur seule échappatoire. Il s'agit, comme le nomme l'auteur, de «l'art du changement ", c'est-à-dire un retournement possible du fil de vie, qui est parfois autant imprévisible qu'inattendu. Le troisième chapitre renvoie à la quête d'humanité, à la recherche de sens à sa vie ou encore à l'adhésion à " une conception élargie du jeu de la vie ". Ces chapitres (le deuxième et le troisième) révèlent des histoires de jeunes qui déambulent dans un monde où il y a perte de sens et qui se demandent comment naviguer dans une société où les repères sont trop souvent inaccessibles puisque leur vie dans la famille et à l'école a été parsemée d'embûches. Au quatrième chapitre, la question qui se pose pour ces jeunes consiste à savoir si la mort est une option. Les témoignages des jeunes évoquent ici des conflits internes qui sont une source d'angoisse dont il faut se débarrasser. Il faut écarter l'ancienne vie pour en imaginer une autre, s'éloigner de la mort pour reconstruire sa vie.

Alors que la première partie du livre exprime « une représentation tragique faite de participations sociales incertaines, voire inhumaines ", la deuxième se caractérise par " un art de vivre ", même si l'existence et l'expérience des jeunes s'ancrent dans un monde qui demeure non accueillant. Le cinquième chapitre rapporte des récits de jeunes qui cherchent à redonner un sens à leur existence et, de cette façon, à se protéger du suicide. Il s'agit pour certains jeunes de devenir le sujet de leur vie (pour Kevin, par exemple, c'est apprendre à aimer la vie). Cela conduit au sixième chapitre où il est question de transformation de l'existence. Les jeunes racontent ici comment elles et ils créent leur vie autrement en se construisant des projets de vie tout en s'éloignant des représentations suicidaires. Nous sommes devant des récits de jeunes qui, après avoir vécu de nombreux drames, recadrent leur vie et assument leur droit d'être qui elles ou ils sont. Enfin, le septième chapitre soulève la question du don, qui permet de cadrer sa vie dans une autre forme d'éthique. Les relations, les liens sociaux et le rapport des jeunes avec le monde se transforment et se manifestent dans leur pratique quotidienne. Il est question pour ces jeunes d'entrer dans un processus d'introspection afin de recréer le sens de leur vie. Les drames que les jeunes vivent et leur trajectoire parsemée d'embûches ne les empêchent 
pas de se faire une place. Paul Grell exprime ainsi cette posture : "Nous sommes face à l'existence simple et forte que la servilité fonctionnelle n'a pas encore détruite. »

L'auteur conclut que la mort volontaire des jeunes est le reflet d'une société dans laquelle ils et elles s'insèrent, une société qui offre des conditions de vie qui parfois sont destructrices. Il rappelle que le suicide n'est pas une question psychologique ou encore pathologique; il suggère qu'il faut surtout se questionner sur le sens de l'acte suicidaire chez les jeunes, à savoir " ce qui, depuis leur intériorité, cherche à s'extérioriser ". Pour lui, le suicide est en ce sens un acte plutôt politique qu'individuel. C'est ainsi qu'il faut interroger davantage "les pensées vivantes ", et c'est ce qui constitue le cœur de ce livre, car tous les récits sont des témoignages de jeunes, filles et garçons, qui se questionnent sur le sens de la vie et de la mort.

Tout au long du livre, l'auteur établit un lien avec la recherche de Durkheim sur la question du suicide et retourne à deux questions essentielles, à savoir si le suicide est considéré comme un problème de santé mentale et si le suicide se transmet d'un jeune à l'autre, renvoyant ici à la question de la contagion. Le suicide est-il un virus, une épidémie? Faut-il alors arrêter d'en parler? Durkheim disait à l'époque : "Ce n'est pas le fait d'en parler, mais c'est la manière dont on en parle. » Pour l'auteur, c'est donc le monde social dans lequel sont menées des vies difficiles qu'il faut interroger, observer et analyser. Mais en l'absence de ce type d'interrogation, voire de cette manière d'en parler, Paul Grell se demande si l'on a «définitivement délaissé l'art de comprendre le sens des gestes graves ».

C'est sans doute pour faire revivre cet art que le livre est marqué par des liens avec la littérature, avec des pensées et des passages qui proviennent d'auteurs tels Kundera, Nelly Arcand et Malraux. Il est aussi parsemé de digressions, de discours parallèles, de mises en exergue de petites histoires se référant à la mythologie grecque, dont l'histoire d'Ariane et de Thésée, et aussi de passages d'auteurs où une autre Ariane est mise en scène. Ces digressions nous disposent à entrer dans les récits de ces jeunes femmes et de ces jeunes hommes, tout en étant sensibles à leurs propos. Paul Grell tente ainsi de faire vivre de l'intérieur l'expérience de ces jeunes. Il nous invite à comprendre comment ces jeunes tiennent parole envers elles-mêmes et eux-mêmes en considérant les conditions de leur société d'appartenance. Ce qui donne forme au vécu et à l'expérience de ces jeunes, ce sont les récits qu'elles et ils en font. Et dans ce livre, dont la qualité de l'écriture est exceptionnelle, Paul Grell réussit très bien à éveiller cette sensibilité et cette humanité. Nous ne pouvons pas refermer ce livre sans vivre nous-mêmes une transformation.

Parlons maintenant un peu de la méthode. L'auteur a donné la parole à quelque 300 jeunes francophones du Nouveau-Brunswick, âgés de 21 à 24 ans. C'est leur discours 
ici qui importe; c'est leur représentation de leur vie, de celle des autres, de leurs proches, parents ou amis, et leur représentation de la vie en général, du monde dans lequel elles ou ils négocient entre la vie digne d'être vécue et des conditions de vie difficiles qui parsèment leur trajectoire. C'est un travail immense qui a été fait ici, un travail d'une grande envergure du point de vue méthodologique. La grande sensibilité de Grell transparaît dans sa manière de traiter les récits et dans la façon dont la parole est mise en avant-scène. Cet expert des récits de vie maitrise l'art de l'analyse et de l'écriture, permettant ainsi de faire sens de l'expérience des jeunes rencontrés. Les récits mettent en lumière ce que les jeunes "savent de la partie locale du monde social où ils vivent". Après ce premier niveau d'analyse, 71 récits ont été retenus pour une deuxième et une troisième entrevue prenant en compte la répartition selon la région, l'origine sociale et le sexe. De ce nombre de récits, 35 ont été sélectionnés pour l'écriture de ce livre et sont représentatifs en quelque sorte de l'ensemble des jeunes interviewés lors de cette recherche. Ces 35 récits ont permis, selon Paul Grell, de restituer « la mosaïque sociale des pratiques en lien avec la problématique du suicide».

Enfin, le sujet qu'aborde ce livre est un sujet très sensible : parler de la mort, et surtout de la mort volontaire des jeunes, n'est pas aisé. L'auteur a réussi, en donnant la parole aux jeunes, à nous amener dans leur univers, à saisir leur fragilité et leur force, à comprendre un peu mieux leur pratique et à apprécier davantage la voix de ces jeunes. Nous ne quittons pas ce livre facilement; il continue de nous habiter bien longtemps après sa lecture. Ce livre est et restera un ouvrage important pour mieux saisir les formes de vie précaires des jeunes d'ici et d'ailleurs. 